\title{
Community Newsletter: COVID-19 and social isolation, camouflaging and first impressions, more on the controversial JADD study
}

\section{BY CHELSEY B. COOMBS}

15 AUGUST 2021

Hello, and welcome to this week's Community Newsletter! I'm your host, Chelsey B. Coombs, Spectrum's engagement editor.

The first study that sparked a lot of conversation on social media was about how COVID-19 and social isolation have affected autistic people's mental health. It was led by Liz Pellicano, professor of educational studies at Macquarie University in Sydney, Australia.

https://twitter.com/liz_pellicano/status/1425056017419669506

In a thread on Twitter, Pellicano wrote that the research team analyzed more than 7,000 minutes of interviews with both autistic adults and parents of autistic children about their experiences.

https://twitter.com/liz_pellicano/status/1425056019068030981

Some participants said they enjoyed having a break from their typical obligations when the pandemic-related lockdowns first began.

But many people also had difficult experiences when they couldn't access schools, doctors or other support networks.

Despite stereotypes that paint autistic people as less social than non-autistic people, participants said the lack of social connections during lockdown took a toll on their mental health. 


\section{Spectrum | Autism Research News}

https://www.spectrumnews.org

https://twitter.com/liz_pellicano/status/1425056026055770121

Tali Aderet-German, a postdoctoral researcher at Aalborg University in Denmark, commented on how the stereotype of non-social autistic people has been "shown to be wrong over and over."

https://twitter.com/DrTaliAG/status/1424253119010070530

Our next thread comes from Hannah Belcher, research associate at King's College London in the United Kingdom, whose new study in the Journal of Autism and Developmental Disorders looked at the relationships among autistic camouflaging, age at diagnosis and non-autistic people's first impressions of autistic people.

https://twitter.com/DrHannahBelcher/status/1422537789707595782

In the study, autistic and non-autistic people took the Camouflaging Autistic Traits Questionnaire and had a recorded conversation with a researcher. Then, 127 non-autistic people watched 10 -second clips of the conversations and recorded their first impressions of the participants. The viewers did not know that any of the participants were autistic.

Even when they were camouflaging, autistic people were rated as making worse first impressions compared with non-autistic people, the team found, and autistic women made better first impressions than autistic men. In addition, autistic people diagnosed at a later age made better first impressions than those diagnosed as young children.

"Coupled with our finding that autistic individuals' deliberate attempts to disguise their condition may not be effective in altering the impression they make on others during social interactions," the researchers write, "this suggests that they should instead be encouraged to embrace an autistic identity and form connections with like-minded people in an authentic way."

Ann Memmott, an associate and 'expert by experience' at the National Development Team for Inclusion in the U.K., tweeted, "We have whole industries set up to teach us how to mask, saying non-autistic people will like us more if we do. No evidence of that."

https://twitter.com/AnnMemmott/status/1422824787211132928

And finally, Sara Luterman's Spectrum piece on the controversial study from the Journal of Autism and Developmental Disorders study that was featured in this newsletter a few weeks back has sparked a lot of conversation in the community.

https://twitter.com/slooterman/status/1425455120519680000

Steve Silberman, author of "NeuroTribes: The Legacy of Autism and the Future of 


\section{Spectrum | Autism Research News}

https://www.spectrumnews.org

Neurodiversity," tweeted, "What on Earth was the Journal of \#Autism and Developmental Disorders thinking?"

https://twitter.com/stevesilberman/status/1425474124927836175

Aimee Grant, senior research officer at Swansea University in Wales, said, "It feels like the editorial process at JADD has broken down."

https://twitter.com/DrAimeeGrant/status/1425523719330480138

Monique Botha, research fellow at the University of Stirling in Scotland, tweeted, "We have had some serious issues trying to report on papers which breach their ethical guidance and COls, including harmful research."

https://twitter.com/DrMBotha/status/1425523520356892677

Finally, don't forget to register for our 31 August webinar with Laurent Mottron, professor of psychiatry at the University of Montreal in Canada, who plans to discuss "a radical change in our autism research strategy."

And we just added a 28 September webinar, featuring Jeremy Veenstra-VanderWeele, professor of psychiatry at Columbia University, who will speak about goals for developing new drugs for autism — and the barriers researchers may encounter.

That's it for this week's Spectrum Community Newsletter! If you have any suggestions for interesting social posts you saw in the autism research sphere, feel free to send an email to me at chelsey@spectrumnews.org. See you next week!

Cite this article: https://doi.org/10.53053/NMFY2942 